\title{
Gradhiva
}

GRADHIV

Revue d'anthropologie et d'histoire des arts

17 | 2013

L'esthétique du geste technique

\section{Du geste technique à la geste musicale}

From labour to song and back again

\section{Marianne Lemaire}

\section{(2) OpenEdition}

Journals

Édition électronique

URL : http://journals.openedition.org/gradhiva/2598

DOI : $10.4000 /$ gradhiva.2598

ISSN : 1760-849X

\section{Éditeur}

Musée du quai Branly Jacques Chirac

\section{Édition imprimée}

Date de publication : 16 mai 2013

Pagination : $50-69$

ISBN : 978-2-35744-049-74

ISSN : 0764-8928

Référence électronique

Marianne Lemaire, « Du geste technique à la geste musicale », Gradhiva [En ligne], 17| 2013, mis en ligne le 27 mai 2013, consulté le 19 avril 2019. URL : http://journals.openedition.org/gradhiva/2598 DOI : 10.4000/gradhiva.2598 


\section{Du geste technique à la geste musicale}

par Marianne Lemaire

Cet article explore les diverses façons dont la musique, lorsqu'elle accompagne une tâche, lui apporte son concours. À partir de quelques exemples de chants de travail empruntés à des cultures diverses et à des contextes économiques différents, il montre que, si la musique est au service du travail qu'elle scande, elle l'est en dehors de toute considération réelle d'efficacité technique et au mépris des fruits qu'il est susceptible de porter; elle soutient une tâche en cours de réalisation dont la finalité est écartée au profit du sens et même de la valeur que les chants s'emploient à lui donner ou, dans le cas du travail servile ou forcé, à lui rendre. De sorte que si le travailler peut être à l'origine du musiquer, il n'en est pas la finalité. 
1. À propos de la pensée de Karl Bücher, voir Hanse 2007 et 2009.

2. André Schaeffner (1980 [1936] : 95 sq.) regrette que Karl Bücher ait limité l'observation aux seuls chants de travail.

II sera indifféremment question ici de la musique vocale ou instrumentale qui accompagne le labeur.

3. Gerald Porter (1995: 276-288) a notamment montré que les chants dont les dentellières anglaises accompagnaient leur tâche jusqu'à la fin du XIxe siècle, loin de pouvoir être envisagés comme des créations musicales fondatrices, puisaient à l'inverse dans divers répertoires préexistants.
"La musique contraste toujours avec ce que la chanson dit; mais elle contraste toujours comme il faut, opposant à la misère humaine une consolation d'avance et comme une vue sur un long déroulement de temps. " Alain, Système des beaux-arts

Que la musique trouve son origine dans le travail est une idée déjà ancienne, qui a compté d'ardents défenseurs. Parmi eux, l'économiste allemand Karl Bücher ${ }^{1}$ (1899) est sans doute celui qui, au début du xx $x^{\mathrm{e}}$ siècle, l'a le mieux formulée. Selon lui, les populations «primitives" n'auraient jamais fait preuve d'indolence mais auraient rencontré des difficultés pour organiser leurs travaux; elles se seraient attachées à donner un rythme à chacun d'eux, à le rendre aussi sonore que possible, et parfois même à lui adjoindre mélodie et harmonie. De telle sorte que, libérées du souci des gestes à accomplir, elles auraient été en mesure de travailler avec entrain tout en développant leur créativité à travers la composition de chants pour les accompagner. Selon Bücher, le travail ne serait donc pas seulement utile à la production de biens nécessaires au groupe social; il serait également à la source de l'art poétique et vocal ${ }^{2}$. On sait cependant aujourd'hui que les chants de travail ne sauraient en aucun cas être considérés comme "premiers ${ }^{3}$ ". Aussi le thème de la relation entre musique et travail est-il plus volontiers l'occasion dans la littérature ethnologique d'envisager, plutôt que ce que la musique doit au travail, ce que le travail doit à la musique et d'explorer les diverses façons dont un chant, lorsqu'il accompagne une tâche, lui apporte son concours. Dans le prolongement de cette perspective, cet article se propose de montrer que la musique est utile à un travail qui, lui, ne l'est pas toujours. À partir de quelques exemples de chants empruntés à des cultures diverses et à des contextes économiques différents, nous allons voir en effet que si la musique est au service du travail qu'elle scande, elle l'est dans le plus grand mépris des fruits qu'il est susceptible de porter; elle soutient un travail en cours dont la finalité est écartée au profit de la valeur et du sens qu'elle s'emploie à lui donner ou même, dans le cas du travail servile et forcé, à lui rendre.

\section{La musique en maître d'œuvre}

Écoutant les chants dont les femmes mossi, au Burkina Faso, entourent leur travail solitaire de mouture du mil, l'ethnologue Junzo Kawada (1998: 112) a le sentiment qu'ils «ne sont pas vraiment faits pour donner un rythme à leur tâche", mais "naissent plutôt du mouvement cadencé de leur corps, simple et prolongé». Certes, la musique, quand elle seconde le travail, prend appui sur lui au point de donner l'impression qu'elle n'en est que le prolongement. Mais si elle ajuste ainsi ses phrases mélodiques et rythmiques aux gestes qui le composent, c'est moins pour le perpétuer que pour lui être utile en proposant une forme de contrôle. La musique se présente en effet comme le maître d'œuvre ou le chef d'orchestre du travail au cours duquel elle intervient. Ainsi, dans les chants qui, en pays sénoufo, au nord de la Côte d'Ivoire, sont exécutés lors du damage du sol des maisons (Lemaire 2009: 111-112), la note la plus basse de l'échelle, dotée d'une valeur longue et accentuée, est toujours mise en correspondance avec le moment où la dame que l'ouvrière vient de soulever s'abat sur le sol, de sorte que l'alternance des notes hautes et basses se confond avec une alternance analogue dans la gestuelle des travailleuses. Par cette sonorisation du geste 


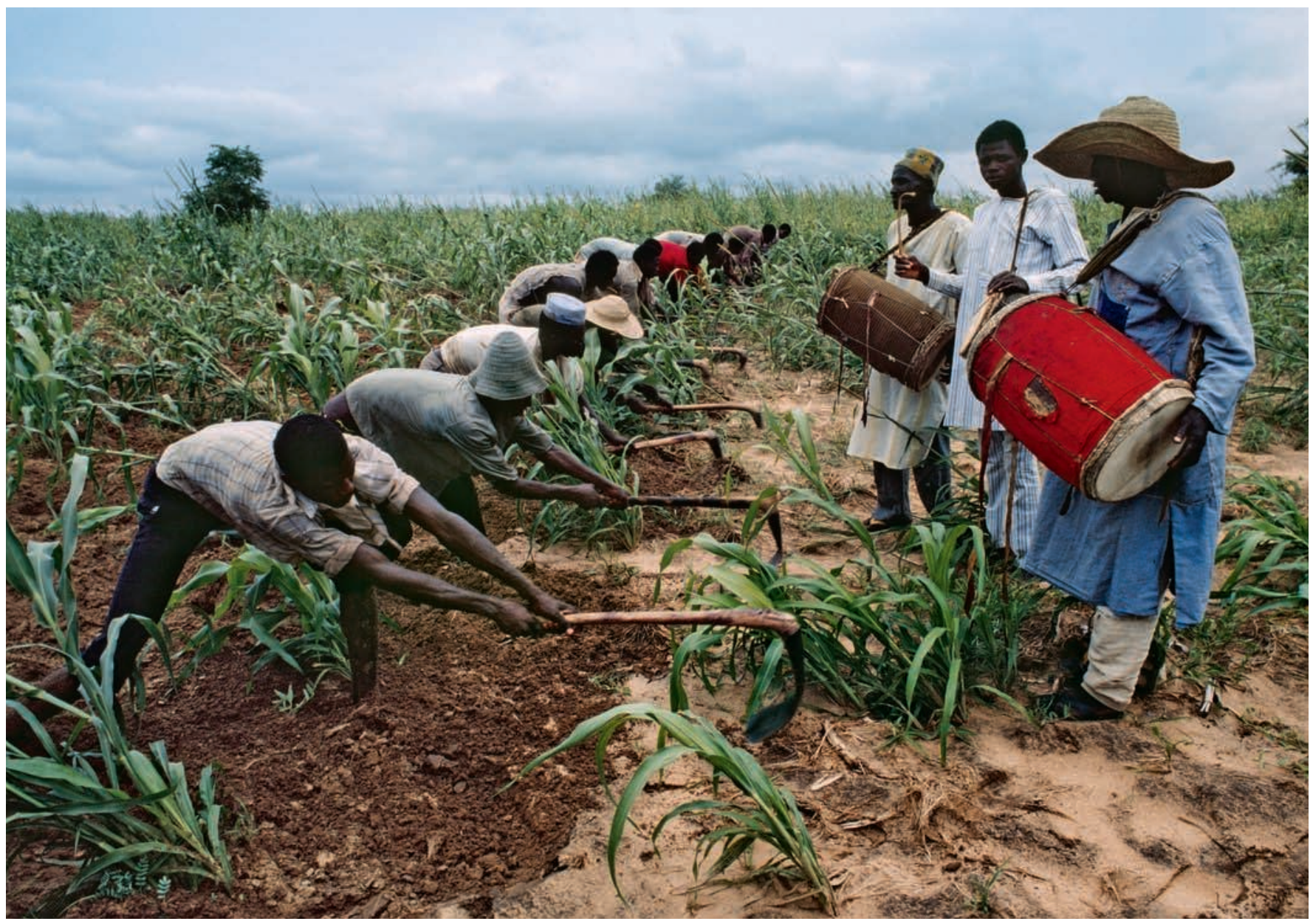

page 50 et ci-dessus

fig. 1

Steve McCurry, Cultivateurs

dans un champ de millet,

Niger, 1986. (C) Steve

McCurry/Magnum Photos. 


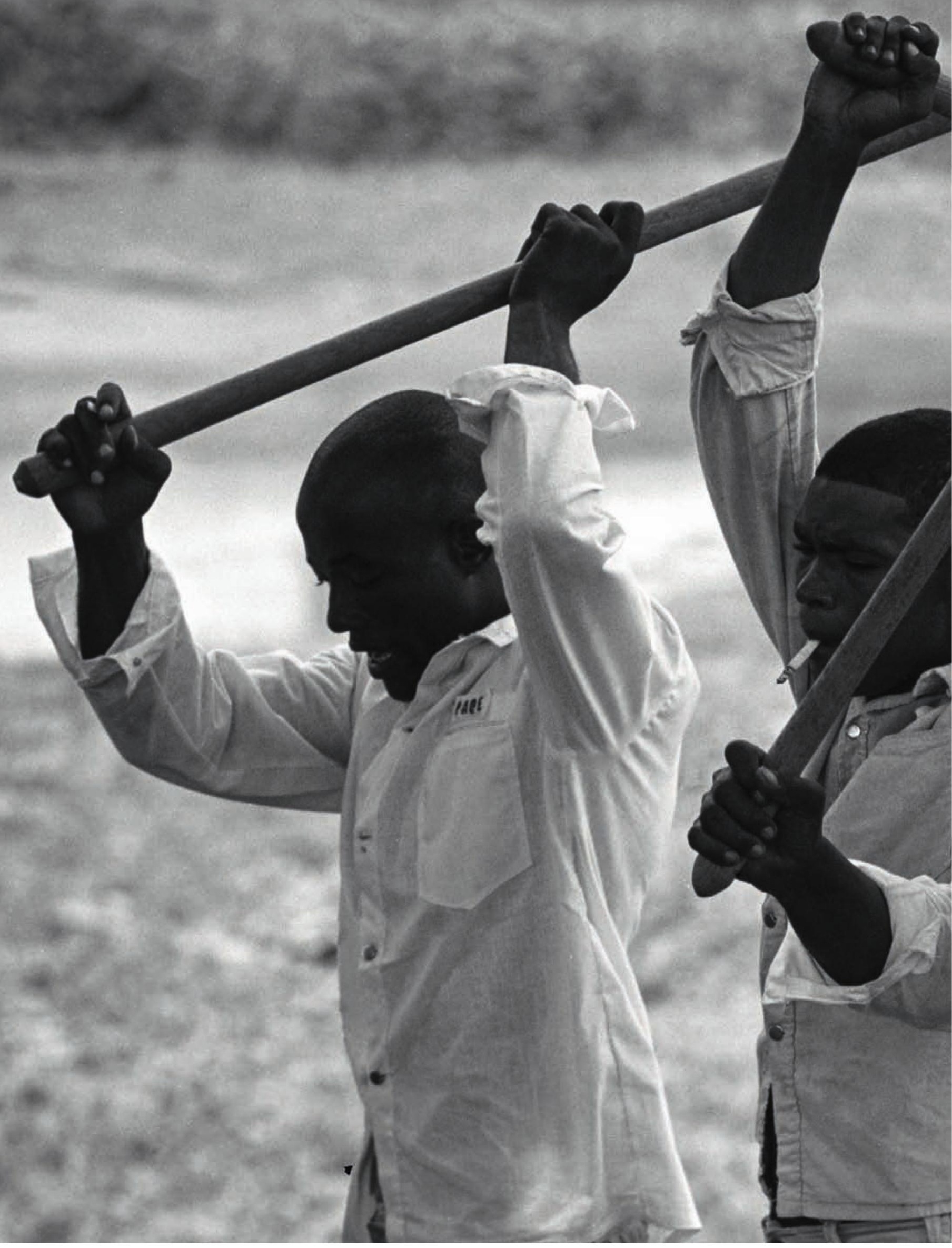




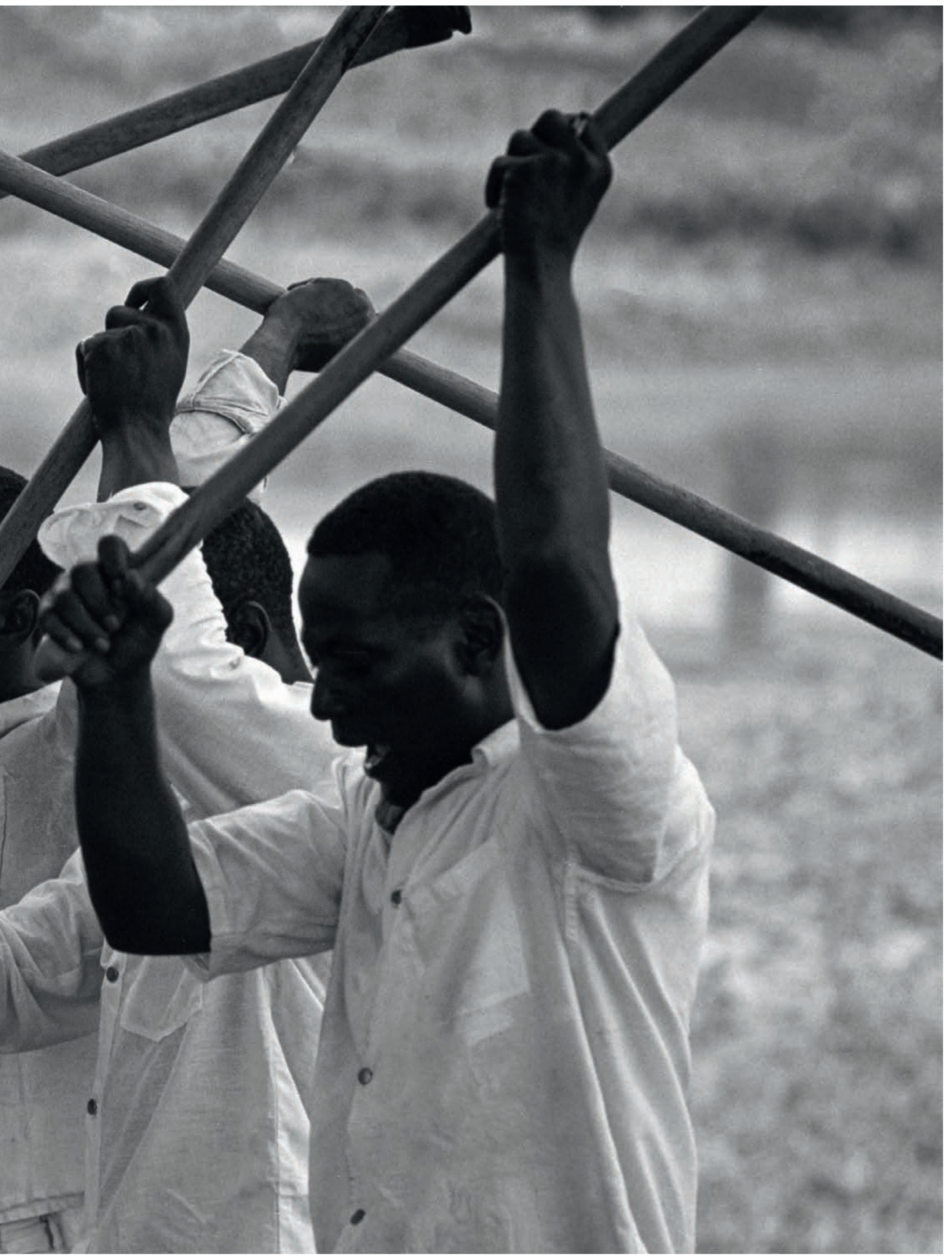




\section{double page \\ précédente}

fig. 2

Bruce Jackson, Travaux de désherbage par Johnny Jackson et son équipe, Texas, Ramsey Prison Farm, 1965. (C) Photo Bruce Jackson.
4. Voir Isabelle Peere (2002) pour les comptines des dentellières brugeoises et Gerald Porter (1994 et 1995) pour celles chantées en Grande-Bretagne.

5. À secourir ainsi le travail, la musique ne sort pas toujours indemne: il arrive en effet que son utilité s'exerce à ses dépens. Ainsi, selon Bruce Jackson (1972: 30-33), les chants de travail aux paroles les plus poétiques et aux mélodies les plus complexes sont aussi ceux dont la fonction utilitaire de rythmer la tâche et de synchroniser les gestes est moindre. Les chants qui accompagnent la cueillette du coton sont ainsi d'une grande richesse, au contraire de ceux qui accompagnent les travaux agricoles au rythme très rapide, ou pire, la tâche dangereuse de couper un arbre à plusieurs.

6. Alan Lomax (2012 [1993] : 337) faisait la même observation dans l'univers carcéral du pénitencier de Parchman, dans l'État du Mississippi : «Fixer exactement le tempo correct, c'est la base de toute prestation musicale réussie, mais, dans les chants de la prison, le tempo avait un rôle bien plus crucial. Le rythme fixé par le meneur du chant indiquait une cadence pratique pour le travail, en lien avec le genre et l'ampleur de la tâche, la météo, les capacités et les sentiments de la brigade."

7. «Dix-neuf longues lignes suspendues devant mon huis,/ Plus vite je travaillerai, plus vite le compte sera bon. » transfiguré en hauteur musicale, la musique lui donne un rythme garant de sa régularité en même temps que de sa synchronisation avec ceux des autres ouvrières. Mais la musique peut plus subtilement encore entourer et accorder entre eux les gestes des travailleurs qui recourent à elle: dans les chants exécutés par les dentellières des ateliers anglais ou belges jusqu'à la fin du $x x^{e}$ siècle, chaque phrase musicale correspondait aux mouvements de boucler une maille et de la maintenir avec une épingle, tandis que son rythme reproduisait en l'accentuant celui engendré par leur alternance ${ }^{4}$.

Au travail, la musique apporte donc non seulement une "parure sonore", mais aussi une "sonorité utile", pour reprendre les belles expressions d'André Schaeffner (1980 [1936]: 96) et Arnold van Gennep 5 (1953: 2681). Et elle remplit d'autant mieux cette fonction qu'elle s'accommode aussi bien d'un tempo lent que d'un tempo rapide. Dans les années 1960, les détenus des prisons texanes confiaient à Bruce Jackson (1972: 17-18) leur préférence pour les chants de travail qu'il était possible d'exécuter dans un tempo lent lorsqu'il s'agissait de dissimuler l'épuisement d'un des leurs ou dans un tempo rapide lorsqu'il importait plutôt de soulager une atmosphère devenue trop pesante ${ }^{6}$.

Mais la musique ne se rend pas seulement utile aux gestes de travail et à leur synchronisation; elle l'est également au procès de travail dans son entier et aux étapes dont il se compose. Aux dentellières, le nombre de vers débité permettait de supputer au fil de leur tâche le nombre de mailles réalisées et donc le moment d'en changer. À cette fin, de nombreux chants faisaient commencer leurs vers par l'énoncé d'un chiffre (Porter 1995: 282):

\section{Nineteen long lines hanging over my door, The faster I work it will shorten my score 7 .}

Les paroles des chants interviennent volontiers pour apporter, au côté de la phrase mélodique et rythmique, leur aide au travail. En pays sénoufo, l'orchestre de xylophones qui, en certaines circonstances, accompagne les travaux agricoles, profère des paroles (Lemaire 2009: 80): la langue sénoufo étant une langue à tons, chaque phrase musicale sous-tend un énoncé verbal qui suit le même schéma tonal et accentuel. Or cet énoncé verbal concerne volontiers l'organisation du travail en cours. S'adressant à l'ensemble des cultivateurs, les xylophonistes leur signifieront par le biais de leurs instruments de musique que le travail peut commencer ou, à l'inverse, qu'il doit s'interrompre, le temps d'une pause ou une fois la tâche achevée (ibid. : 88-90):

Arrêtez-vous, ou quelqu'un va s'évanouir

Nous allons arrêter les xylophones et rentrer au village.

Ils pourront également diriger autoritairement les travailleurs vers un espace jusque-là négligé par eux et les enjoindre à le cultiver au plus vite: 
Le répertoire des détenus texans recueilli par Jackson tout comme celui des détenus du pénitencier de Parchman qu'Alan Lomax et son père enregistrèrent dans l'État du Mississippi entre 1933 et 1947 comptaient également des chants dont les paroles avaient trait au travail en cours et permettaient qu'il se déroule au mieux. Tel chant alertait ainsi à la ronde, au cours du travail consistant à couper des arbres à la hache, de la chute prochaine de l'un d'eux ${ }^{8}$ :

Oh well my timber gettin' limber and it's fallin' down, Oh well my timber gettin' limber and it's fallin' down, I done a-warned you, done told you that it's fallin' down, I done a-warned you, done told you that it's fallin' down. Well if it hits you, don't you holler, man, it's fallin' down, Well if it hits you, don't you holler, Lord, it's fallin' down. Oh well my timber start to crackin' and it's fallin' down, I say my timber start to crackin' and fallin' down.

Paroles et mélodies, on le voit, participent activement à la bonne marche de la tâche qu'elles secondent. Cependant, si la musique renforce la régularité et l'organisation du travail, elle lui est dans le même temps salutaire dans la mesure où elle rompt sa monotonie et le récrée. De sorte qu'après avoir été utile en renforçant sa répétition, la musique se rend utile au travail en la camouflant.

\section{Le travail en jeu}

Bücher l'avait bien relevé et amplement commenté: la musique agrémente le travail et réjouit ceux qui le réalisent. Son rythme les vivifie, et ses mélodies leur procurent du plaisir. Encore faut-il ajouter à cela que la musique accompagne rarement le travail sans s'accompagner elle-même du jeu. Ainsi, leurs chants donnaient de l'entrain aux dentellières et les retenaient de s'endormir les soirs d'hiver dans la mesure où leurs paroles étaient souvent fantaisistes, associant propos facétieux, jeux de mots et recherches d'assonances ou d'allitérations (Porter 1995: 280-281; Peere 2002). D'autres chants, très narratifs, réclamaient quant à eux d'être joués autant que chantés, après que les rôles eurent été distribués comme à la courte paille à l'aide des fuseaux. L'une des pièces énonçait d'ailleurs clairement sa fonction essentielle de distraction (Peere 2002: 117):

Et, pendant le travail chantent

Nos bouches toutes ensemble

Pour contenir notre nonchalance

Par des airs plaisants.

Les situations de travail les plus difficiles pouvaient ainsi être égayées à l'aide des propos tenus dans les chants auxquels elles donnaient lieu. Lomax évoque par exemple le «rire gargantuesque» qui semblait s'élever du chœur des prisonniers de Parchman, après que le soliste eut énoncé "une phrase de conclusion salée, calculée pour faire sourire tout le monde» (Lomax 2012 [1993]: 336).
8. "Fallin' down», in Jackson 1972: 222-228. Voici la traduction de l'extrait: "Oh ouais, ma poutre fléchit et elle tombe, (bis)/J't'l'avais bien dit, j't'avais dit qu'elle allait tomber. (bis)/Ouais, si ça te tombe dessus, ne braille pas, mec, elle tombe,/ Ouais, si ça te tombe dessus, ne braille pas, Seigneur, elle tombe./Oh ouais ma poutre commence à craquer et à tomber,/ J'te dis que ma poutre commence à craquer et à tomber. ” 
À moins que l'amusement introduit par la musique au cœur du travail ne soit gestuel plutôt que verbal. Dans le cas des chants à damer sénoufo (Lemaire 2009: 112-114), le moment du refrain voit les ouvrières se livrer tour à tour au jeu de projeter leur outil de travail dans les airs, de taper dans leurs mains libérées de l'outil et de le rattraper au vol juste à temps pour pouvoir le frapper sur le sol au moment précis de la prochaine pulsation. Ce faisant, elles réalisent une véritable figure rythmique qui vient alors enrichir le chant auquel elle doit son apparition.

Cette dimension de jeu que la musique apporte volontiers au travail prend parfois la forme plus institutionnalisée d'une compétition entre les travailleurs. Comme le signale Isabelle Peere, certains vers des chants exécutés par les dentellières laissent à penser qu'elles se livraient entre elles à une course de vitesse (Peere 2002: 119):

Celle qui a placé la première et la dernière épingle remporte le tout, du tout au tout.

On peut certes imaginer que cette compétition dans le travail puisse avoir lieu en l'absence de toute musique pour encadrer l'une et l'autre. Mais dans bien des contextes, musique et compétition semblent étroitement liées. Ainsi, parmi les détenus texans, le statut de «meilleur travailleur » dans les tâches effectuées en musique, telles la cueillette du coton ou l'abbatage des arbres, n'était pas dissociable de celui de meneur et donc de «meilleur chanteur » des chants qui les rythmaient: les deux qualités étaient attribuées au même individu (Jackson 1972: 35). De même, les xylophonistes sénoufo qui encouragent les cultivateurs, lorsqu'ils jouissent de la réputation de figurer parmi les meilleurs instrumentistes, bénéficient volontiers aussi de celle de figurer parmi les meilleurs travailleurs à la houe. Leurs chants, en outre, soutiennent et organisent tout autant la compétition à laquelle le travail donne lieu que le travail lui-même. Car s'ils s'adressent souvent à l'ensemble des cultivateurs, les xylophonistes ont un interlocuteur privilégié en la personne du meilleur d'entre eux, le tegbanwi, "celui qui est fort à la houe». En la circonstance des concours très formalisés qui opposent les cultivateurs de deux villages distincts lors du travail de buttage des ignames, deux orchestres de xylophones sont présents, qui s'adressent chacun au champion de travail agricole de leur village. Postés au bord du champ, ils s'emploient toute la journée durant à l'encourager en décrivant sa progression et en vantant ses multiples qualités (Lemaire 2009: 91):

L'homme est petit sur le corps, il fait vraiment un joli travail.

Mais les xylophonistes savent que la louange des qualités physiques du champion ne constitue pas un encouragement suffisant. Ils s'appliquent aussi à éveiller en lui la douleur morale qui doit attiser son désir de vaincre, faisant ainsi office de ressort principal de la rivalité sur laquelle repose le concours.

Tout se passe comme si l'introduction de la musique dans un contexte de travail favorisait celle de la compétition, et avec elle l'atmosphère de fête dont l'une et l'autre ne peuvent tout à fait se priver. C'est bien une fête qui entoure le concours de travail agricole sénoufo, comme elle entoure 


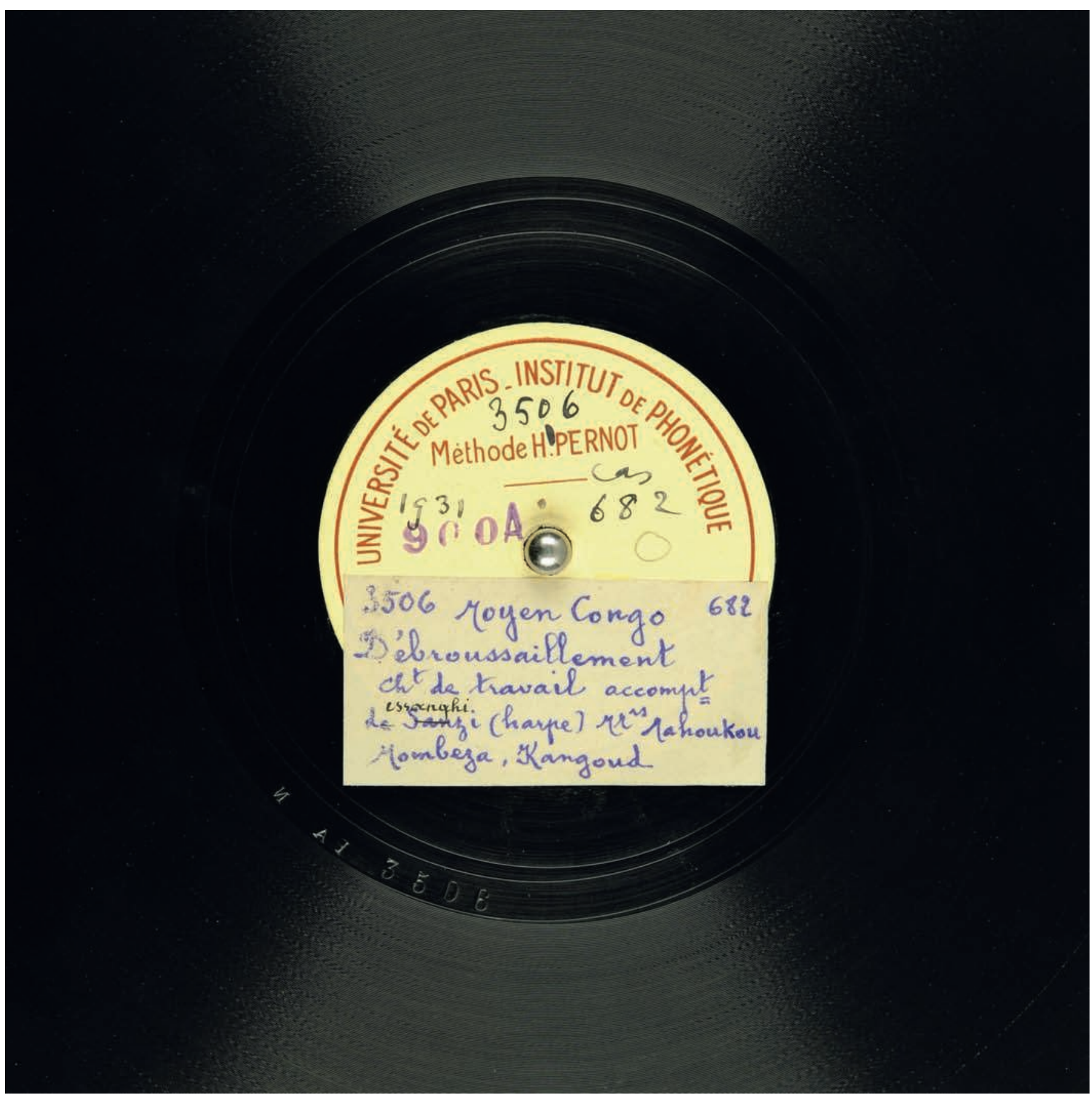

fig. 3

Rondelle du disque 78 tours Débroussaillement: chant de travail. MM. Monbeza et Mahoukou, chant; accompagnement de pluriarc (luth à archet, kondé) et de battements de mains,

Paris, Exposition coloniale internationale, 1931.

Université de Paris, musée de la Parole et du Geste. (C) Bibliothèque nationale de France, Département audiovisuel. 
9. «Toutes ensemble un jour ensoleillé,/Nos coussins brillent comme les bourgeons en mai,/Nos doigts sont agiles, nos bobines sont petites,/

Et voilà vous savez tout. " la tâche consistant à damer le sol des maisons ou celle, en Bretagne jusque dans les années 1930, de fouler la terre en dansant, au son de musiciens ou de chanteurs, pour aménager ou restaurer l'aire où les céréales étaient annuellement battues au fléau (ibid. : 49-51). Derrière la musique et la danse se profilaient, au cours de ces «grandes journées» qui voyaient venir des habitants de différents villages pour faire une «aire neuve» (al leur nevez), des séances de lutte ou des jeux sportifs pour reposer les travailleurs, ainsi qu'un repas copieux pour les sustenter. De sorte que musique et travail réunis semblent toujours avoir une façon bien à eux de reconsidérer la distinction entre le labeur et la fête, et aussi bien entre l'utile et l'agréable. Mais revenons-en pour l'heure à la musique, et examinons cette autre fonction qu'elle en vient à endosser dès l'instant où elle scande un travail, qui consiste à permettre à ceux qui le réalisent de se l'approprier pleinement.

\section{Un travail à soi}

Nul doute que le chant dont ils prennent le soin de s'assister ne permette aux travailleurs de s'approprier, en tout premier lieu, leur espace de travail. Ainsi, les chants de rodage entonnés par le laboureur poitevin l'aident à "créer de l'intimité», selon les termes de Michel de Lannoy (1994: 108), dans un espace par trop inhospitalier et dépeuplé. De la même façon, en pays sénoufo, les chants à damer ont le souci de rendre familier un espace qui, s'il est destiné à être domestique, ne l'est pas encore devenu, tandis que les chants de xylophone se chargent d'humaniser un lieu de culture à la frontière de l'univers dangereux de la brousse. À cet égard, le chant de travail évoque un peu la «ritournelle» telle que la concevaient Gilles Deleuze et Félix Guattari (1980: 383-384): une mélodie qui retient toujours « un peu de terre » en son sein, distribue des repères et territorialise.

Mais c'est aussi la tâche elle-même que la musique permet aux travailleurs d'investir et de s'approprier. À cette fin, celle-ci renforce préalablement les liens entre les membres du collectif de travail et leur sentiment de former une communauté en recourant à un vocabulaire connu d'eux seuls, en évoquant un savoir-faire commun ou simplement en gravant dans leur esprit l'image du groupe soudé qu'ils forment (Porter 1995: 280):

We all together on a sun shiny day,

Our pillows they shine like the blossom in May,

Our fingers are lissom, our bobbins are small,

And now I have told you the truth of it all ${ }^{9}$.

Une fois le groupe rassemblé, la musique est en mesure d'accueillir l'expression d'une souffrance commune à l'ensemble des travailleurs. Tel est le cas des chants des jeunes femmes et des enfants soumis au travail minutieux et exténuant de la dentelle, strictement encadré par des associations d'acheteurs dans le cas de l'industrie villageoise anglaise ou par des «maîtresses» dans le cas des écoles-ateliers de Bruges. Quelques chants, il est vrai, participaient au durcissement de la discipline de travail, faisant par exemple planer la menace d'une punition sur quiconque briserait le rythme ou relèverait la tête avant d'avoir compté vingt mailles (ibid. : 285): 


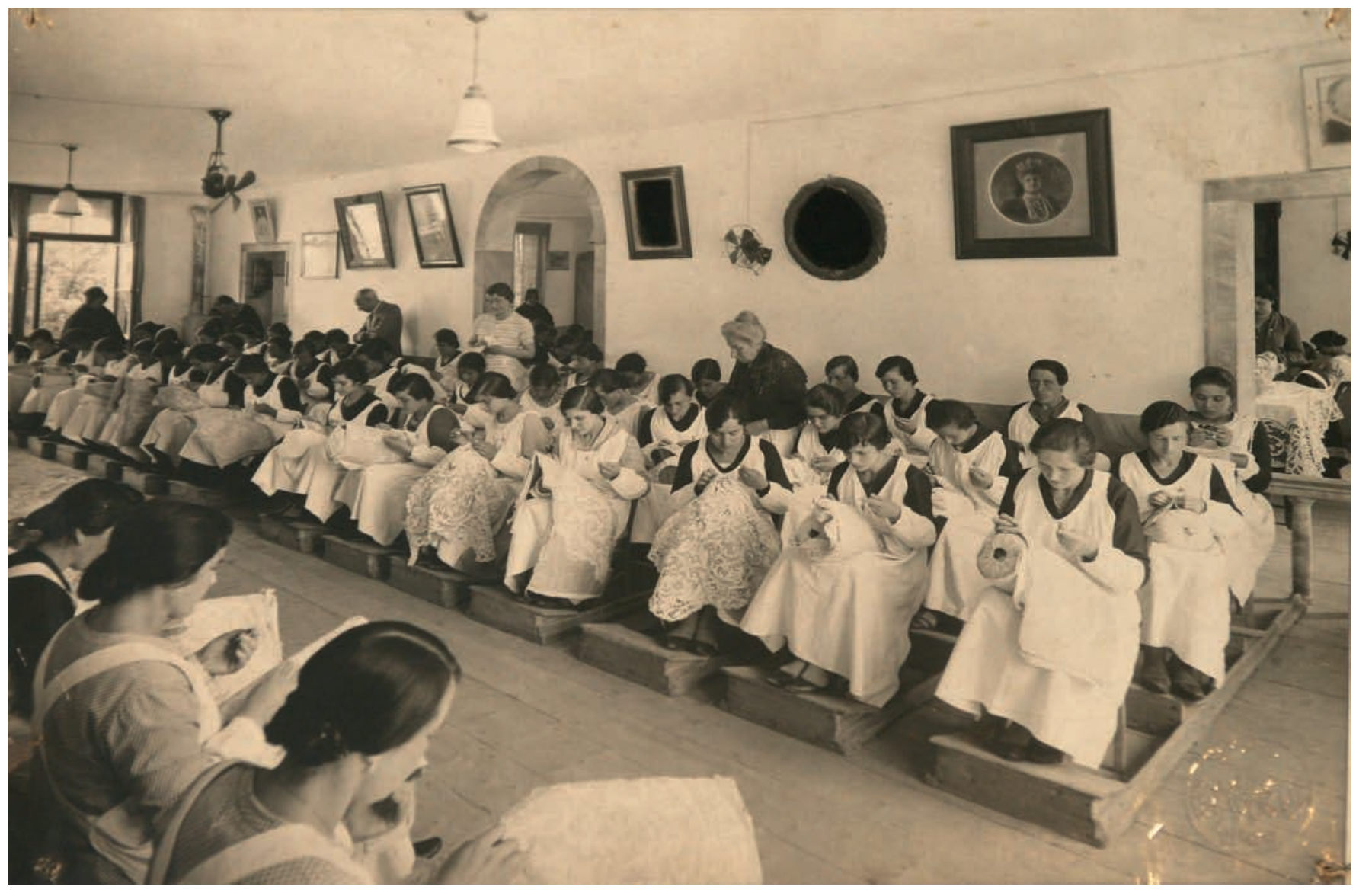

fig. 4

Photographe italien anonyme,

Dentellières à Burano, Venise.

(c) The Bridgeman Art Library. 
10. "Un cheval pour conduire ma dame-/Ne doit pas regarder ailleurs jusqu'à ce que vingt soit compté,/ Suspends-la pendant une demi-heure,/Et coupe-la comme une fleur."

11. "You tell the truth about how you feel ", confie un prisonnier à Bruce Jackson (1972: 18).

12. "Make a longtime man feel bad ", in Jackson 1972 : 77-78.

\begin{abstract}
A horse to carry my lady about -
Must not look off till twenty are out.

Hang her up for half an hour

Cut her down just like a flower ${ }^{10}$.
\end{abstract}

Mais les chants de dentellières s'attachaient avant tout à dénoncer leurs difficiles conditions de travail. La souffrance pouvait y être exprimée aussi bien sur le ton de la plainte ou de la revendication que sur celui de I'humour, comme dans ce chant qui met ironiquement en scène un échange entre une maîtresse et un acheteur brugeois (Peere 2002: 122):

Monsieur, mes enfants ne veulent pas travailler,

Qu'est-ce que j'en fais?

Mettez-les tous dans un lieu obscur,

Et donnez-y leur de l'avoine et de l'eau de vaisselle

Et un peu à manger.

D'une souffrance l'autre, le pas est rapidement franchi: les travailleurs, quelle que soit leur tâche, investissent volontiers leurs chants de l'expression d'une douleur personnelle ou collective extérieure au travail. Les femmes mossi, au moment de moudre le mil, formulent ainsi «des reproches à l'égard de leur mari, du ressentiment vis-à-vis des parents qui les ont mariées à tel époux ou une apologie de leur famille d'origine" (Kawada 1998: 112). Dans un registre similaire, les chants à damer des femmes sénoufo dépeignent pour bon nombre d'entre eux un personnage féminin qui, éloigné de ses parents utérins et rejeté par les parents de son époux, est contraint à l'isolement (Lemaire 2009: 117-118):

Toi maintenant tu restes seule,

Tu piles dans la grande cour, pareille à un mortier dans la cour.

La solitude peut aussi être la conséquence, non plus de l'éloignement, mais de la perte définitive de proches:

Toi, prends le récipient du malheur, il te sied bien,

Toi, qui chargera l'eau sur ta tête?

Les chants de travail des détenus du Texas et du Mississippi constituaient également un lieu privilégié de l'expression d'une profonde souffrance. Un peu comme aux femmes mossi autorisées, le temps que dure leur tâche, à proférer des plaintes et des reproches, les chants de travail permettent rien moins aux détenus que de dire la vérité sur la manière dont ils se sentent 11. Comme le souligne Lomax (2012 [1993]: 344), "ces chants sondaient les profondeurs de leur désespoir, tout en affirmant leur détermination à endurer ". La solitude et la perte de l'être aimé en même temps que celle de la liberté en étaient dès lors les principaux thèmes ${ }^{12}$ :

Roberta, let your hair grow long,

Let it grow so long till it drags the ground.

Make a longtime man feel bad.

Make a longtime man feel bad, 
I don't get no letter, I don't hear from home, Make a longtime man feel bad ${ }^{13}$.

De nombreux chants de prisonniers texans exprimaient également un profond sentiment d'injustice ${ }^{14}$ :

If you see my mother, partner, tell her pray for me, I got life on the river, yeah, never will go free, never will go free. They 'cuse me a murder,

Never harmed a man, never harmed a man ${ }^{15}$.

Le même sentiment d'injustice animait les détenus du pénitencier de Parchman, dont un chant rappelait avec humour que la seule faute dont ils s'étaient rendus coupables était d'être nés dans le Mississippi et d'y être restés (ibid. : 331):

\section{It ain't but the one thing I done wrong \\ I stayed in Miss'ippi just a day too long ${ }^{16}$.}

Ainsi, grâce à la musique, qui accueille les sentiments les plus intérieurs et les plus douloureux des travailleurs, ceux-ci s'approprient leur travail. Une nécessité d'autant plus impérieuse que les travaux imposés aux détenus du Mississippi ou du Texas portent à leur paroxysme les dimensions de pénibilité et de contrainte que renferme toute tâche. Nul doute que les chants des prisonniers, qui appartenaient à une tradition remontant à l'arrivée des premiers esclaves en Virginie en $1631^{17}$, leur restituaient un semblant de liberté ou tout au moins de libre-arbitre dans un contexte qui les en privait totalement: "The songs change the nature of the work by putting the work into the workers's framework rather than the guards'. By incorporating the work with their song, by, in effect, co-opting something they are forced to do anyway, they make it theirs in a way it otherwise is not ${ }^{\mathbf{1 8}}$." (Jackson 1972: 30) Lomax (2012 [1993] : 344) remarque également, concernant les détenus de Parchman, que leurs chants figuraient parmi les seules choses qu'ils possédaient en propre, et à propos desquelles ils étaient en mesure de faire des choix: "Les chants étaient les leurs - ceci était leur art, recréé tel qu'ils l'interprétaient, en suivant un meneur qu'ils avaient choisi et qui se devait de leur plaire ou bien serait remplacé par un autre. Le meneur tissait une nouvelle trame de couplets à partir d'une réserve commune qui, n'appartenant qu'à eux, déclarait parfaitement leurs sentiments et les différenciait de leurs gardiens et du monde libre - lequel les punissait si cruellement. »

Mais les prisonniers n'exerçaient pas leur liberté par le seul choix de chanter, ou de chanter leur souffrance; ils l'exerçaient également par d'autres choix proprement musicaux. Non seulement ces work songs et ces chain gang songs ${ }^{19}$ permettaient d'exprimer la pénibilité du travail, mais ils permettaient aussi, grâce à leurs paroles allusives ou ironiques, leur forme responsoriale (appels/réponses), leurs notes à la tonalité instable (blue notes), leurs superpositions rythmiques (binaire/ternaire), leurs registres vocaux distordus à l'image presque exacte des corps s'éreintant sous le labeur et la chaleur, de transformer les conditions d'existence
13. "Roberta, laisse pousser tes cheveux,/Laisse-les pousser jusqu'à ce qu'ils traînent par terre./Ça fait qu'un taulard de longue peine se sent mal./Ça fait qu'un taulard de longue peine se sent mal,/J'ai pas de lettre, j'sais rien de la maison,/Ça fait qu'un taulard de longue peine se sent mal. »

14. "If you see my mother », in Jackson 1972: 79-80.

15. «Si tu vois ma mère, mon pote, dis-lui de prier pour moi,/J'ai pris perpète en taule. ouais, j'serai plus jamais libre, plus jamais libre./ Ils m'accusent de meurtre,/ J'ai jamais fait de mal à un homme, jamais fait de mal à un homme. »

16. "C'est le seul truc que j'ai pas fait comme il faut/ Je suis resté dans le Miss'ippi rien qu'un jour de trop. »
17. Seuls les détenus africains-américains des prisons américaines chantaient au cours de leur travail.

18. "Les chants changent la nature du travail en le plaçant dans la perspective des travailleurs plutôt que dans celle des gardiens. Investir le travail de leurs chants, adhérer de fait à quelque chose qu'ils sont de toute façon obligés de faire, c'est leur manière de faire leurs ce qui, autrement, ne l'est pas. »

19. Chants de groupes enchaînés. "Au cours du XIX ${ }^{e}$ siècle, le coût du système carcéral et le besoin de main-d'œuvre amènent les autorités judiciaires du Sud [des États-Unis] à mettre en place la location de détenus" (Levet 2003: 130) à des entreprises privées. Ce système a survécu jusqu'à l'aube des années 1960 et a été réactivé en Alabama au cours des années 1990. 


\section{4}

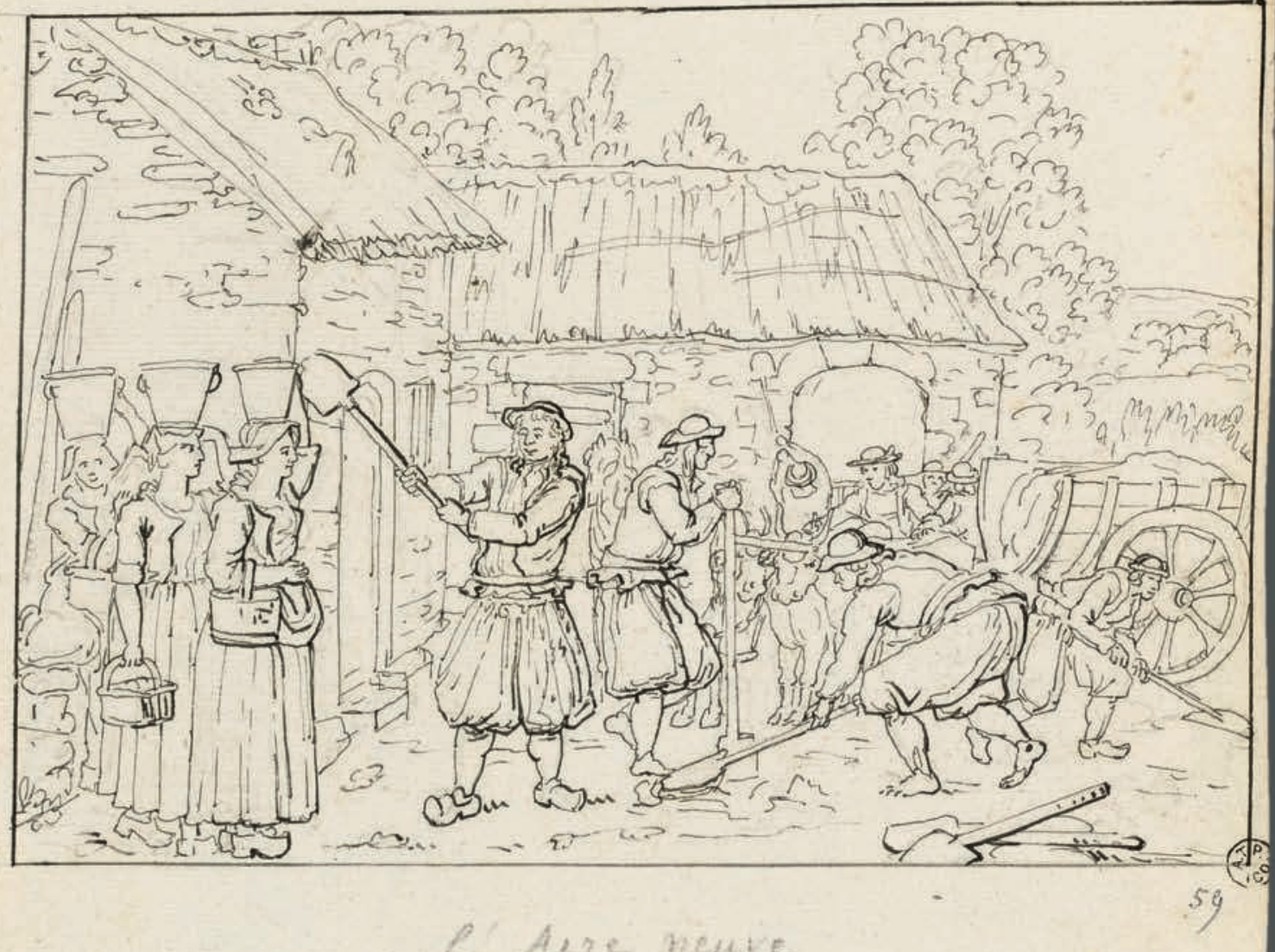

E Alre peute 
de ceux qui les chantaient en conditions de délivrance ${ }^{20}$. Aussi ces chansons de travail, ces mélopées, ces cantilènes émises «du fond d'une fosse profonde", comme le disait la chanteuse de gospel Mahalia Jackson, portent-elles en elles le flottement rythmique et mélodique, et même harmonique et sémantique qu'elles transmettront par la suite au blues et au jazz, et dans lequel Jacques Réda (2009: 43) voit la «culbute émancipatrice» à même de changer la cadence en rythme, tandis que Jean Jamin et Patrick Williams (2010: 27) y perçoivent la trace d'une «esthétique du non " et la possibilité de l'esquive 21 .

\section{Le travail en soi}

Les chants de travail, on le voit, rendent bien des services à la tâche qu'ils accompagnent. Ils soutiennent le geste technique, entourent celui-ci d'une gaieté qui n'occulte pas néanmoins la dimension de souffrance qui lui est associée et dont l'expression permet à ceux qui le réalisent de se l'approprier. Observateurs et travailleurs sont d'ailleurs unanimes pour reconnaître les effets bénéfiques de la musique sur le travail. Les chants de xylophone, de l'avis des cultivateurs, les emplissent de courage et d'ardeur en «levant leurs mains», et leur permettent de soutenir l'effort dans la durée en «asseyant leur cœur» (Lemaire 2009: 90, 110); ceux des détenus leur donnent la possibilité de «relâcher la tension» qui les habite (Jackson 1972: 17), et leur rendent leur travail et leur condition un peu plus supportables; ceux des dentellières semblent avoir stimulé leur zèle et soutenu leur concentration (Porter 1995: 280); danse et musique de l'aire neuve, quant à elles, ont pour effet reconnu et recherché par les Bretons de «casser la fatigue» (Guilcher 1963: 17).

Il est également bien souvent entendu dans la littérature consacrée aux chants de travail que, dans la mesure où la musique scande une tâche, elle favorise sa productivité. Selon les historiens, les planteurs américains auraient ainsi admis les vertus proprement économiques des chants de leurs esclaves, et donc renoncé à les leur interdire (Jamin et Williams 2001: 338). Bücher dresse quant à lui le tableau d'un rendement décuplé grâce à la présence de musiciens pour stimuler le zèle des travailleurs (Hanse 2007: 212). Et Alexandre Bouët (1986 [1838]), un homme de lettres du milieu du $\mathrm{XIX}$ siècle, de comparer les frais engagés par le propriétaire de la ferme en vue de la fête de l'aire neuve à un «investissement », et à un investissement soumis aux règles de la rationalité économique puisque largement «amorti » par le résultat obtenu. Mais Peere (2002: 116) a raison d'adopter une formulation plus prudente en affirmant que «s'ils [les chants] étaient tolérés pendant le travail, c'est sans doute qu'ils avaient un effet favorable sur la productivité de l'atelier, notamment en accordant toutes les dentellières à travailler au même rythme».

II n'est certes pas impossible de concevoir que la musique puisse avoir des effets bénéfiques sur les produits du travail qu'elle soutient. Mais c'est alors comme par accident, tant la relation que la musique noue avec le travail auquel elle se joint semble être d'une autre nature. II est peu probable que les détenus texans, au moment où ils entonnaient leurs chants, songeaient à des résultats économiques qui leur échappaient. Toute leur préoccupation était à l'inverse de rendre supportable un travail
20. Voir les analyses et réflexions de LeRoi Jones (1968 [1963] : 29 sq.) sur les chants de travail des esclaves africains devenus, par la force des choses et du temps, des «esclaves américains".

21. "Probablement, la condition servile des Noirs transplantés de force aux Amériques, et leurs modes de réaction, qui ne pouvaient être que suggestifs, allusifs, décalés sont-ils pour beaucoup dans cette amphibologie de la syncope, dans ce renversement des points d'appui qui, de la marche, cherchait à faire autre chose qu'un pas cadencé ou un moyen de se rendre au travail. La cueillette du coton ou le partage de ses balles, l'enfoncement des tire-fonds sur les traverses des rails de chemin de fer, le fauchage des talus bordant les routes par des prisonniers noirs entravés..., tout cela qui devait se produire en cadence, à l'instar d'un moteur à vapeur ou à explosion, mais dont tout hoquet ou raté était annonciateur de panne, donnait prise au moindre boitement, au moindre glissement, au moindre balancement, au moindre claquement de pointe ou de talon, qui, plutôt qu'à un grippage, préludaient alors à une émancipation de la marche et du geste moins par rapport à leur fonction qu'à leur but : aller travailler, certes contraint, forcé, mais pas à la manière d'une troupe de soldats s'exposant à la décimation. La cadence, ne serait-ce qu'étymologiquement, implique la chute; la syncope permet l'esquive. " (Jamin et Williams 2010: 27)

\footnotetext{
ci-contre

fig. 5

Olivier Perrin, L'Aire neuve, vers 1810, extrait d'un ensemble de dessins édités sous le titre Galerie bretonne chez Isidore Pesron, Paris, 1835-1838, gravés par Réveil avec des textes de Louis Auguste Maréchal et Alexandre Bouët. Marseille, musée des Civilisations de l'Europe et de la Méditerranée (MuCEM). (C) RMN-Grand Palais (MuCEM)/Franck Raux.
} 
en cours de réalisation, en l'investissant d'une part d'eux-mêmes à travers l'expression de la souffrance qu'ils enduraient et l'exercice du peu de liberté dont ils disposaient encore.

II ne fait pas non plus de doute que les chants de xylophone sénoufo exécutés lors des concours de cultivateurs à la houe ont un tout autre objectif que d'augmenter leur productivité. Bien au contraire, la rivalité avec laquelle la musique est en étroite relation tout au long du concours aurait plutôt l'effet inverse: loin de provoquer une émulation susceptible d'accélérer ou d'accroître la production, la concurrence, si elle n'est pas modérée, se concentre sur les seuls champions au point de décourager tous les autres cultivateurs et de ralentir le rythme de travail. Pas plus que les chants des détenus texans ou mississippiens, les chants de xylophone ne sont tournés vers la finalité des gestes qu'ils accompagnent.

L'exemple des forgerons mossi illustre également cette vocation de la musique à enrichir le temps du travail plutôt qu'à en accroître les fruits. Autrefois, les forgerons réalisaient, en actionnant leurs soufflets, des motifs rythmiques complexes par lesquels ils s'échangeaient des formules de salutation ou de remerciement, des informations sur l'avancée de leur travail ou des plaisanteries. Ces motifs rythmiques pouvaient également donner lieu à des concours de virtuosité entre les travailleurs qui les maîtrisaient le mieux. Tel n'est plus le cas aujourd'hui: les forgerons les plus âgés regrettent amèrement que les nouvelles générations se contentent de réaliser des motifs rythmiques élémentaires, et ceci en raison d'un souci de rentabilité qui œuvre aux dépens de la part d'amusement que le travail comportait en leur temps: "Mais en ce qui concerne les jeunes", disent les anciens interrogés par Lidia Calderoli (2010: 92), «ils cherchent seulement à attiser le feu, ils ne cherchent pas à s'amuser».

Il est aussi intéressant de noter que, dans un article où il se penche sur «l'efficacité», ne serait-ce que symbolique, de musiques associées à la pratique de la chasse chez les Pygmées, concluant qu'ils conçoivent leur activité musicale comme une "activité de survie", Gilbert Rouget (2004) n'examine que des répertoires exécutés non pas pendant le travail cynégétique proprement dit, mais avant ou après celui-ci. En somme, tout se passe comme si la musique ne pouvait être investie d'un souci d'efficacité que lorsqu'elle précède la tâche, à moins qu'elle ne lui succède. Mais qu'elle l'accompagne au moment même de son déroulement, et elle ne le fait alors que pour la soulager en lui donnant tout son sens et sa valeur.

\section{Conclusion}

La musique n'intervient donc pas pour orienter les travailleurs vers la finalité productive du travail, mais les reconduit au contraire vers le travail en lui-même. En redoublant son caractère répétitif et cyclique, en adhérant à ses gestes, elle met en évidence les dimensions d'effort, de tension et d'endurance qui le composent. Elle le situe ainsi définitivement du côté du travail tel que le concevait Hannah Arendt (1983 [1958]: 123-230): un travail qui, au contraire de l'œuvre, ne réifie pas le monde en le peuplant d'objets solides et durables, ne prend jamais fin, et voit sa finalité se confondre avec l'effort que l'ensemble du corps humain doit fournir lors de sa réalisation 


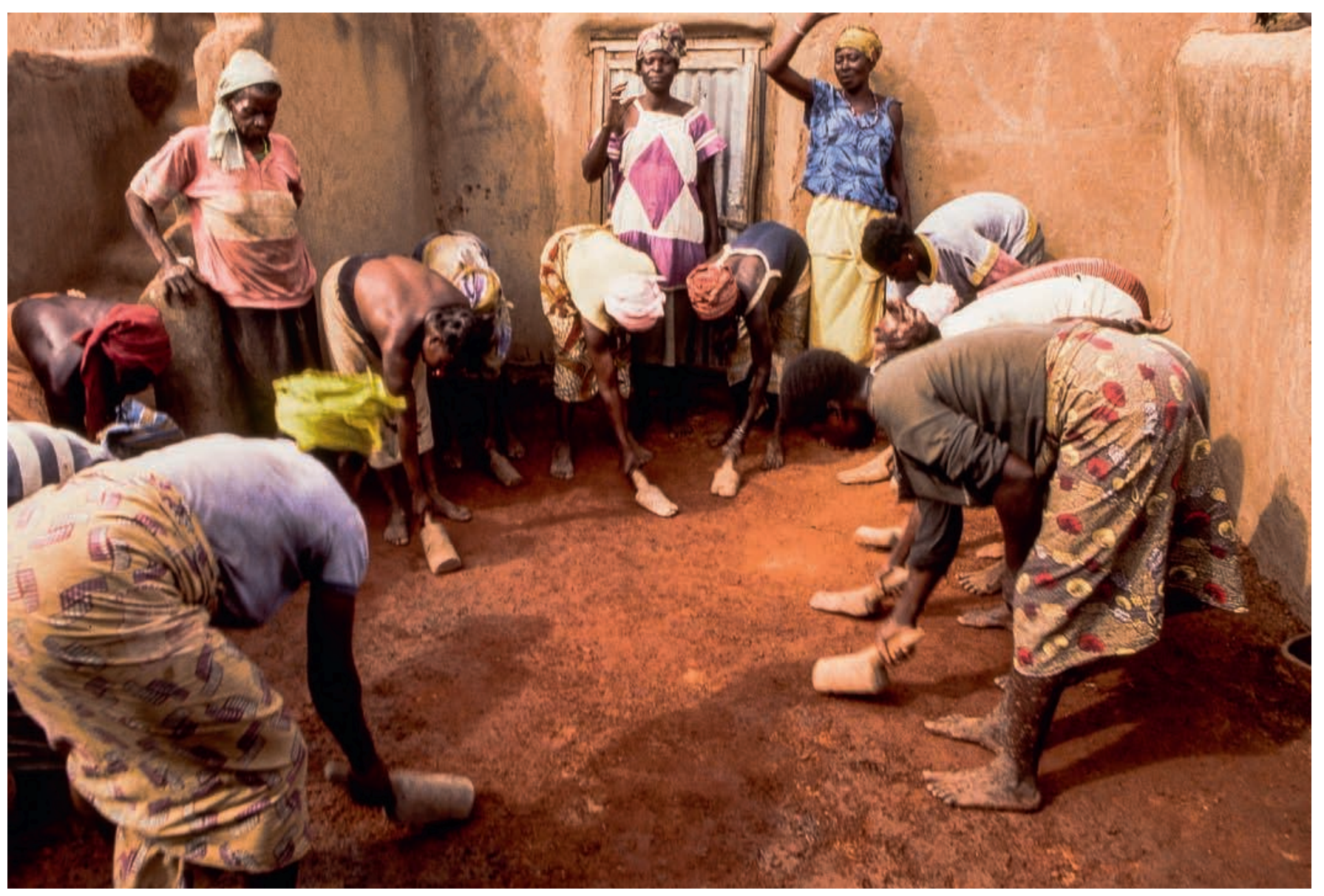

et de sa nécessaire répétition. Cependant, la musique ne se contente pas d'imiter le travail; elle s'emploie également à l'embellir, l'enrichissant de chants, de jeux, de danses, de divertissements, mais aussi de réflexions sur la souffrance, la privation de liberté, la solitude ou la mort ainsi que sur la possibilité d'y échapper. Nourri par la musique, le travail acquiert une nouvelle profondeur et permet à ceux qui le réalisent de vivre pleinement, dans sa durée, le moment de l'effort. Au-delà des oppositions classiques entre le beau et l'utile, les moyens et les fins, la musique et le travail forment alors, ensemble, une véritable geste musicale. fig. 6

Damage du sol chez les Kassena, Burkina Faso, 1995. () Georges Courrèges (www.flickr.com/photos/ courregesg). 


\section{Arendt, Hannah}

1983 (1958) Condition de l'homme moderne. Paris, Calmann-Lévy.

\section{Bouët, Alexandre}

1986 (1838) Breiz-Izel ou la vie des Bretons de l'Armorique. Paris, Seghers.

\section{Bücher, Karl}

1899 Arbeit und Rhythmus. Leipzig, Teubner.

\section{Calderoli, Lidia}

2010 Rite et technique chez les forgerons moose du Burkina Faso. Forger, apaiser, soigner. Paris, L'Harmattan.

\section{Deleuze, Gilles et Guattari, Félix}

1980 Mille plateaux. Paris, Éditions de Minuit.

\section{Guilcher, Jean-Michel}

1963 La Tradition populaire de danse en Basse-Bretagne. Paris, Mouton.

\section{Hanse, Olivier}

2007 Rythme et civilisation dans la pensée allemande autour de 1900. Rennes II, thèse de doctorat.

2009 "À la recherche du "travail joyeux". La théorie de Karl Bücher et son influence sur le mouvement du rythme ", Le Texte et l'Idée 24 69-89.

\section{Jackson, Bruce}

1972 Wake up Dead Man Afro-American Worksongs from Texas Prisons. Cambridge, Harvard University Press.

\section{Jamin, Jean et Williams, Patrick}

2001 "Glossaire et index des musiciens de jazz ", L'Homme 158-159: 301-338.

2010 Une anthropologie du jazz. Paris, CNRS Éditions.

\section{Jones, LeRoi \\ 1968 (1963) Blues People. \\ Paris, Gallimard.}

\section{Kawada, Junzo}

1998 La Voix. Étude d'ethnolinguistique comparative. Paris, Éditions de l'École des hautes études en sciences sociales.

\section{Lannoy, Michel (de)}

1994 "La voix dans son espace. Chants de labour en bocage poitevin ", in Jacques Coget (éd.), L'Homme, l'animal et la musique. Saint-Jouinde-Milly, Famdt Éditions: 104-113.

\section{Lemaire, Marianne}

$\mathbf{2 0 0 0}$ "Damer la terre", in Jacques Coget (éd.), L'Homme, le minéral et la musique. Saint-Jouin-de-Milly, Modal: 38-57.

2009 Les Sillons de la souffrance. Représentations du travail en pays sénoufo (Côte d'Ivoire). Paris, CNRS Éditions-Éditions de la Maison

des sciences de l'homme

("Chemins de l'ethnologie»).

\section{Levet, Jean-Paul}

2003 Talkin' That Talk. Le langage du blues et du jazz. Paris, Éditions Kargo.

\section{Lomax, Alan}

2012 (1993) Le pays où naquit le blues. Saint-Sulpice-La-Pointe, Les Fondeurs de Briques.

\section{Peere, Isabelle}

2002 "Comptines de dentellières brugeoises (1730-1850): entre travail, école et jeu, colère et prière", Acta Ethnographica Hungarica 47(1-2): 111-126.

\section{Porter, Gerald}

1992 The English Occupational Song. Stockholm, University of Umea.

1994 " "Work the Old Lady out of the Ditch ": Singing at work by English lacemakers ", Journal of Folklore Research 31(1-3) : 35-55.

1995 " "Wee'l keepe our fingers playing": Women's work songs and the appropriation of tradition ", in James Porter (éd.), Ballads and Boundaries. Narrative Singing in an Intercultural Context. Los Angeles, UCLA, Department of Ethnomusicology and Systematic Musicology: 276-288.

\section{Réda, Jacques}

2009 Battement. Montpellier, Fata Morgana.

\section{Rouget, Gilbert}

2004 "L'efficacité musicale: musiquer pour survivre. Le cas des Pygmées », L'Homme 171-172: 27-52.

\section{Schaeffner, André}

1980 (1936) Origine des instruments de musique. Introduction ethnologique à l'histoire de la musique instrumentale. Paris, Éditions de l'École des hautes études en sciences sociales.

\section{Van Gennep, Arnold}

1953 Manuel de folklore français contemporain, t. I, vol. VI, Paris, Éditions Picard et Cie. ci-contre

Steve McCurry, Cultivateurs dans un champ de millet, Niger, 1986 (C) Steve McCurry/Magnum Photos.

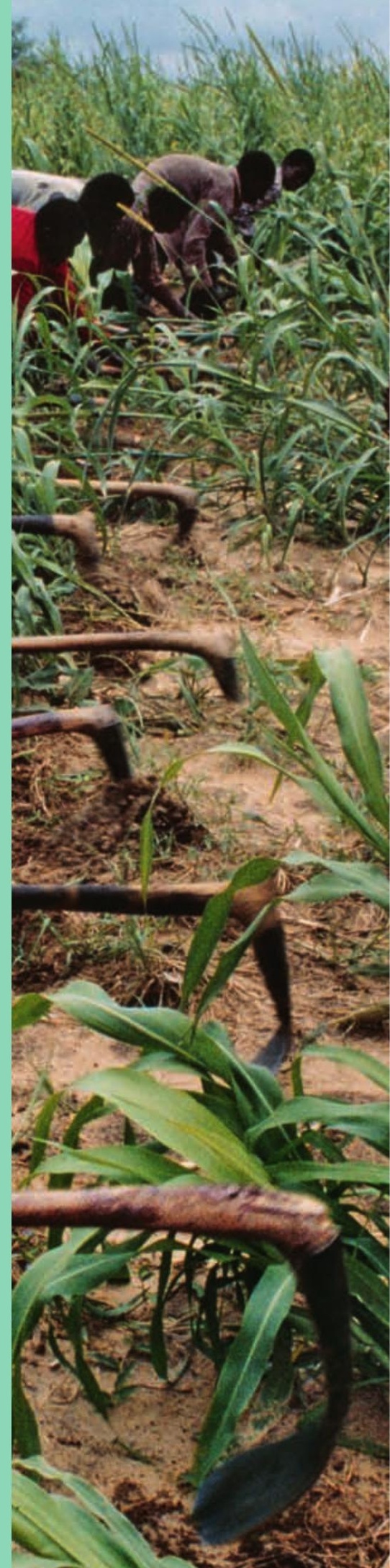




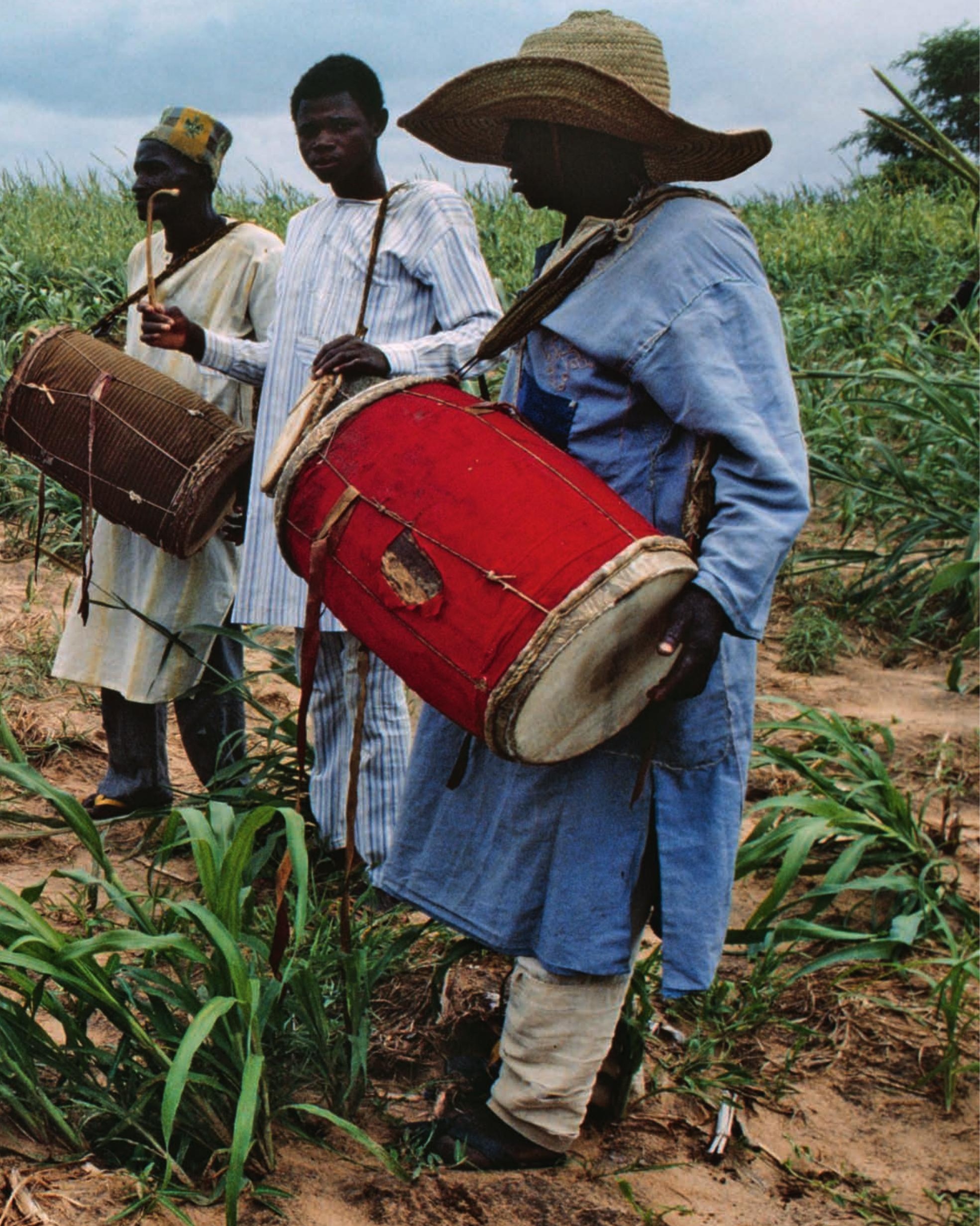

